\title{
INFLUENCE OF POLYETHYLENE OXIDE CONTENT ON SOME CHARACTERISTICS OF PLA/CS FILMS LOADING NIFEDIPINE
}

\author{
Nguyen Thuy Chinh", Nguyen Thi Thu Trang, Tran Thi Mai, Do Van Cong, \\ Mai Duc Huynh, Tran Huu Trung, Vu Viet Thang, Thai Hoang, Nguyen Vu Giang
}

\author{
Institute for Tropical Technology, Vietnam Academy of Science and Technology, \\ No 18, Hoang Quoc Viet, Cau Giay, Ha Noi, Viet Nam \\ "Email: ntchinh@itt.vast.vn or thuychinhhn@gmail.com
}

Received: 10 April 2017; Accepted for publication: 1 October 2017

\begin{abstract}
This work mentions the effect of polyethylene oxide (PEO) content on some characteristics and properties of polylactic acid (PLA)/chitosan (CS) films loading nifedipine (NIF). The films were prepared by solution method with various PEO content in the range of 0 to $10 \mathrm{wt} \%$ in comparison with total weight of both PLA and CS. The water contact angle, droplet size values and Field Emission Scanning Electron Microscopy (FESEM) images of the films were used to investigate their morphology and hydrophobicity. The obtained results show that the hydrophobic property of the PLA/CS/NIF films is improved by the presence of PEO. Besides, the PLA/CS/NIF films containing PEO have tighter structure and water absorbed ability less than those of the PLA/CS/NIF films. The FESEM images of PLA/CS/NIF films with and without PEO before and after 28 hours of drug release testing indicated that NIF were released from these films. The PLA/CS/NIF films with and without PEO are hydrolyzed with weight loss from 22.8 to $90.1 \%$ corresponding to 2 to 28 days of immersing in $\mathrm{pH} 7.4$ solution, respectively.
\end{abstract}

Keywords: polyethylene oxide, compatibility, nifedipine, PLA/CS, hydrolysis.

\section{INTRODUCTION}

Poly lactic acid (PLA) is one of popular biopolymers because it has biodegradation ability and can be recycled under the effect of enzymes and microorganisms. As one of environmentally friendly polymers, PLA is widely used in many different fields such as textile fibers, packaging production and especially in the medical field [1]. Its advantages are high tensile strength and hardness large, potential biocompatibility and biodegradation.

Chitosan (CS) is a polysaccharide which insoluble in water as well as almost organic solvents, however it can be dissolved in some diluted acid solutions. Chitosan and its derivatives exhibit many good properties, for example, antifungal and antibacterial ability, high selfbiodegradable [2 - 3]. Therefore, chitosan and its derivatives can be used in many fields such as pharmaceutical, biomedical, agricultural, industrial, water treatment and environment protection. 
Nifedipine (NIF) is a calcium antagonist drug belonging to the nitro-dihydropyridine group, used for anti-hypertensive, anti-angina and treatment of Raynaud disease [4]. NIF has some disadvantages such as poor solubility in water, short half-life and rapid release rate. Therefore, in order to control the effectiveness and the release rate of NIF in treatment, it is necessary to use suitable drug carriers from natural resource. Among them, PLA and CS are suggested as two potential polymers thanks to their good characteristics and the ability of controlling drug release rate through the formation of the physical interactions between the PLA, CS and drug. However, PLA is a hydrophilic polymer while CS is a hydrophobic polymer; and polymer blends of PLA/CS have poor compatibility. Hence, using compatibilizers is an effective solution for improving compatibility and dispersion ability between PLA and CS. Polyethylene oxide (PEO) has chosen as a compatibilizer for PLA and CS due to safety for human body and the C-O groups in PEO can interact with the $(-\mathrm{C}=\mathrm{O}),(-\mathrm{OH}),\left(-\mathrm{NH}_{2}\right)$ groups in the PLA, CS and NIF molecules $[5,6]$. The obtained PLA/CS films using PEO may have more closely structure and the release rate of NIF from the polymer blends of PLA/CS will be controlled easily.

The solution method is one of the most common methods applied to manufacture the polymer system carrying drugs with some advantages: simple manipulation; the reaction occurs in solution so the reaction temperature can be controlled. This method has been used by some authors in the apothecary of composites loading NIF and some other drugs [7 - 9]. A number of works of PLA/CS composites loading some drugs such as paclitaxel, anthraquinone, lamivudine has been published [5, 10-13]. In our previous studies, the PLA/CS films loading NIF has been prepared successfully by solution method [14]. The SEM images showed that the size of dispersed phase (CS and NIF) in PLA matrix is in the range of $100 \div 300 \mathrm{~nm}$. The slight shift of wavenumber for some groups such as $\mathrm{C}=\mathrm{O}, \mathrm{C}-\mathrm{O}, \mathrm{OH}, \mathrm{NH}_{2}$ belong to PLA, CS, NIF in the FTIR spectrum of PLA/CS/NIF film confirmed that PLA, CS and NIF were interacted with each other. The crystallinity relative degree of PLA/CS/NIF film decreased as compared to PLA/CS film. Additionally, the characteristics, structure, morphology and degradation ability in the different $\mathrm{pH}$ solutions of the PLA/CS films using PEO as a compatibilizer were presented in our previous works $[6,15]$. The FTIR and DSC analysis results showed that NIF was carried by PLA/CS/PEO nanocomposites and PLA, CS, NIF are better interacted and more compatible when using PEO. CS was dispersed more regularly with smaller size in PLA matrix in the presence of PEO. The in-vitro NIF release studies were carried out in $\mathrm{pH} \mathrm{2,6.8} \mathrm{and} 7.4$ solutions corresponding to the $\mathrm{pH}$ of stomach, colon and duodenum regions in human body for 8 hours, respectively. The NIF release content in different $\mathrm{pH}$ solutions is in the order: $\mathrm{pH} 2>\mathrm{pH} 6.8>\mathrm{pH} 7.4$, and increases when rising NIF loading content. However, the influence of PEO content on the hydrophobicity, water absorption ability and morphology of the PLA/CS/NIF/PEO films after drug release testing has not been investigated. Therefore, this study presents the effect of various PEO content on some above properties and morphology of the PLA/CS/NIF films.

\section{EXPERIMENTAL}

\subsection{Materials}

Poly lactic acid (PLA) in grain form with degree of hydrolyzing of $99 \%$, density of 1.25 $\mathrm{g} / \mathrm{cm}^{3}$, viscosity of $\sim 2.0 \mathrm{dL} / \mathrm{g}$, averaged molecular weight (Mw) of 260,000 , polydispersity index (PDI) of 1.5; CS with degree of deacetylation of $77 \%$, viscosity of $1220 \mathrm{cP}$, molecular weight of $1,61 \times 10^{5}$. PEO with Mw 100,000 , glass temperature of $-67^{\circ} \mathrm{C}$; PDI value of $1.02 \div 1.12$; and NIF in yellow powder form, purity $\geq 98 \%$ were used. The above chemicals were provided by 
Sigma-Aldrich Co., USA. Dichloromethane (DCM), acetic acid $\left(\mathrm{CH}_{3} \mathrm{COOH}\right) 99.5 \%$, ethanol $\left(\mathrm{C}_{2} \mathrm{H}_{5} \mathrm{OH}\right)$ were analytical reagents which have been used without further purification.

\subsection{Fabricating PLA/CS films loading NIF with and without PEO by solution method}

PLA and NIF were dissolved in $12 \mathrm{~mL}$ DCM solven ( $1^{\text {st }}$ cup). The mixture of CS and PEO were dissolved in $12.5 \mathrm{~mL} 1 \%$ acetic acid solution ( $2^{\text {nd }}$ cup). Two solutions in the cups were then stirred separately using magnetic stirrer for 2 hours until a homogeneous solution was formed. After that, mixing two solutions was carried out using ultrasonic stirring for 5 minutes before magnetic stirring for 7 minutes. This process was repeated by 3 continuous times. The solutions were put into a petri dish and left to evaporate for 32 hours. Then, these films were dried in the vacuum for 8 hours at $30-35{ }^{\circ} \mathrm{C}$ for obtaining PLA/CS/NIF films. The obtained films have thick-ness around $1 \pm 0.2 \mathrm{~mm}$. The ratio of components in films and abbreviation of the different films are listed in Table 1.

Table 1. The abbreviation of the different PLA/CS/NIF films: F: film, P: PLA, C: Chitosan, N: Nifedipine, P0, 2, 4, 6, 8 and P10 corresponding to PEO content 0, 2, 4, 6, 8 and $10 \%$, respectively.

\begin{tabular}{|l|c|c|c|c|}
\hline Sample & PLA content $(\%)$ & CS content $(\%)$ & NIF content $(\%)$ & PEO content (\%) \\
\hline FPCN & 72.0 & 18.0 & 10 & 0 \\
\hline FPCP2N & 70.4 & 17.6 & 10 & 2 \\
\hline FPCP4N & 68.8 & 17.2 & 10 & 4 \\
\hline FPCP6N & 67.2 & 16.8 & 10 & 6 \\
\hline FPCP8N & 65.6 & 16.4 & 10 & 8 \\
\hline FPCP10N & 64.0 & 16.0 & 10 & 10 \\
\hline
\end{tabular}

\subsection{Characterization of the PLA/CS/NIF films with and without PEO}

\subsubsection{Water contact angle (WCA)}

The water contact angle of the PLA/CS/NIF films with and without PEO was determined by static sessile drop method on the SEO Phoenix-150 meter at the Institute of Energy Science, Vietnam Academy of Science and Technology (VAST). Distilled water was placed randomly on chosen positions at the surface of the films. The WCA was calculated by average value of 5 points per specimen and repeated 3 times.

\subsubsection{Field emission scanning electron microscopy (FESEM)}

Morphology of PLA/CS/NIF films with and without PEO before and after drug release studies was observed and imaged on the scanning electron microscopy radiated field device (FESEM S- 4800, Hitachi, Japan) at the National Institute of Hygiene and Epidemiology and Institute of Materials Science, VAST.

\subsubsection{Water absorption determination}

Water absorption of PLA/CS/NIF films with and without PEO was determined as follows: all film specimens were weighed with same weight and immersed in distilled water. After each 
interval (2, 4, 6 and 8 hours), one of 4 film specimens was taken and dried to remove water completely from the film. And then, the weight number was weighed and noted exactly. All steps were performed 3 times per specimen and taken the average value. Water absorption was calculated by equation below:

$$
\alpha=\left(\mathrm{m}_{\mathrm{t}}-\mathrm{m}_{\mathrm{o}}\right) / \mathrm{m}_{\mathrm{o}} \times 100 \%
$$

where $m_{o}$ and $m_{t}$ are the weight of film before and after immersion $(\mathrm{g})$, respectively.

\subsubsection{Determination of weight loss of PLA/CS/NIF films with and without PEO in $p H 7.4$} solution

Determination of weight loss of the films immersed in $\mathrm{pH} 7.4$ solution was calculated based on the change of sample weight after hydrolysis according to following equation:

$$
\mathrm{m}=\mathrm{m}_{\mathrm{r}} / \mathrm{m}_{\mathrm{i}} \times 100 \%
$$

where $\mathrm{m}$ is the weight loss of samples $(\%), \mathrm{m}_{\mathrm{i}}$ is the initial weight of samples $(\mathrm{g})$ and $\mathrm{m}_{\mathrm{r}}$ is the weight of samples after hydrolysis $(\mathrm{g})$.

\section{RESULTS AND DISCUSSION}

\subsection{The hydrophobic/hydrophilic properties of PLA/CS/NIF films with and without PEO}

Figure 1 presents the value of water contact angle (WCA) of PLA/CS/NIF films with and without PEO. It is clear that WCA values of all investigated films are higher than $90^{\circ}$ corresponding to hydrophobic materials [16]. Moreover, the WCA value of PLA/CS/NIF/PEO films is higher than that of the PLA/CS/NIF film. This is explained by the role of PEO as a compatibilizer for PLA, CS, and NIF due to hydrogen bonding and dipole - dipole interactions between of $\mathrm{C}=\mathrm{O}, \mathrm{C}-\mathrm{O}, \mathrm{OH}, \mathrm{NH}$ groups in PLA, CS, and $\mathrm{NH}, \mathrm{C}-\mathrm{O}, \mathrm{C}=\mathrm{O}, \mathrm{N}=\mathrm{O}$ groups in NIF. Therefore, it limits water absorption into the surface of these films resulting in the increase of their hydrophobicity [16]. The films containing 6 and $8 \%$ PEO have the largest WCA value corresponding to the most closely structure which are expressed by morphology of the PLA/CS/NIF films with and without PEO (see Section 3.2).

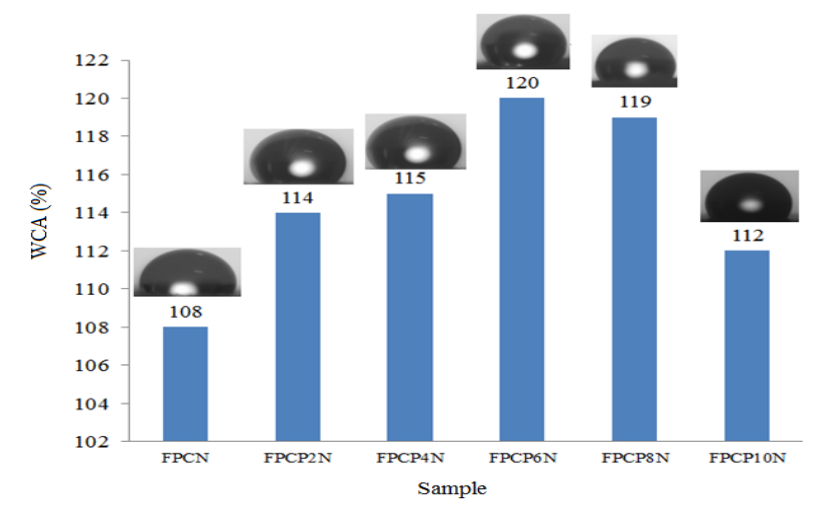

Figure 1. Water contact angle (WCA) of PLA/CS/NIF films with and without PEO.

\subsection{Morphology of the PLA/CS/NIF films with and without PEO}

Figure 2 shows FESEM images of PLA/CS/NIF films with and without PEO. It is obviously that the structures of all films are heterogeneous with two phases: PLA matrix phase 
and the dispersed phase containing CS and NIF. The presence of PEO may help CS and NIF better disperse and stick with each other and form smaller nanoparticles due to physical interactions as aforementioned. The change in the content of PEO also influences on dispersion of CS and NIF in PLA matrix. In FPCN films, CS is dispersed in the PLA with the size about $10 \div 20 \mu \mathrm{m}$ and agglomerated into cluster particles while NIF is dispersed in PLA with smaller particle size, about $1 \mu \mathrm{m}$. At $2 \mathrm{wt} \%$ PEO (FPCP2N film), CS particle is dispersed in the range of $5 \div 20 \mu \mathrm{m}$ in PLA matrix and the particle size of NIF is reduced to $200 \div 500 \mathrm{~nm}$. At $4 \mathrm{wt} \%$ PEO (FPCP4N film), CS and NIF are dispersed in the PLA phase with the size of $1 \div 5 \mu \mathrm{m}$ and $200 \div 500 \mathrm{~nm}$, respectively. In particular, CS and NIF are found with the smallest size, only about $1 \div 5 \mu \mathrm{m}$ and $50 \div 100 \mathrm{~nm}$ in FPCP6N film (using $6 \mathrm{wt} \%$ PEO). However, in FPCP8N and FPCP10N films (using 8 and $10 \mathrm{wt} \%$ PEO), CS is dispersed irregularly and agglomerated to larger particle clusters, about $10 \mu \mathrm{m}$ or larger size. This indicates that the PLA/CS/NIF film using $6 \mathrm{wt} \%$ PEO has more evenly structure and size of CS and NIF dispersed in PLA smaller than the films containing other content of PEO. This may be due to small content of PEO is not enough to improve the interoperability between PLA, CS and NIF. Nevertheless, with the larger PEO content, residual content of PEO after creating bonding and interaction with PLA, CS, NIF can cause the phase separation in the structure of films leading to decrease compatibility of PLA, CS, PEO and NIF. The dispersion of NIF in PLA/CS/6 wt\% PEO film can be observed in FESEM images at different magnification (Figure 3).
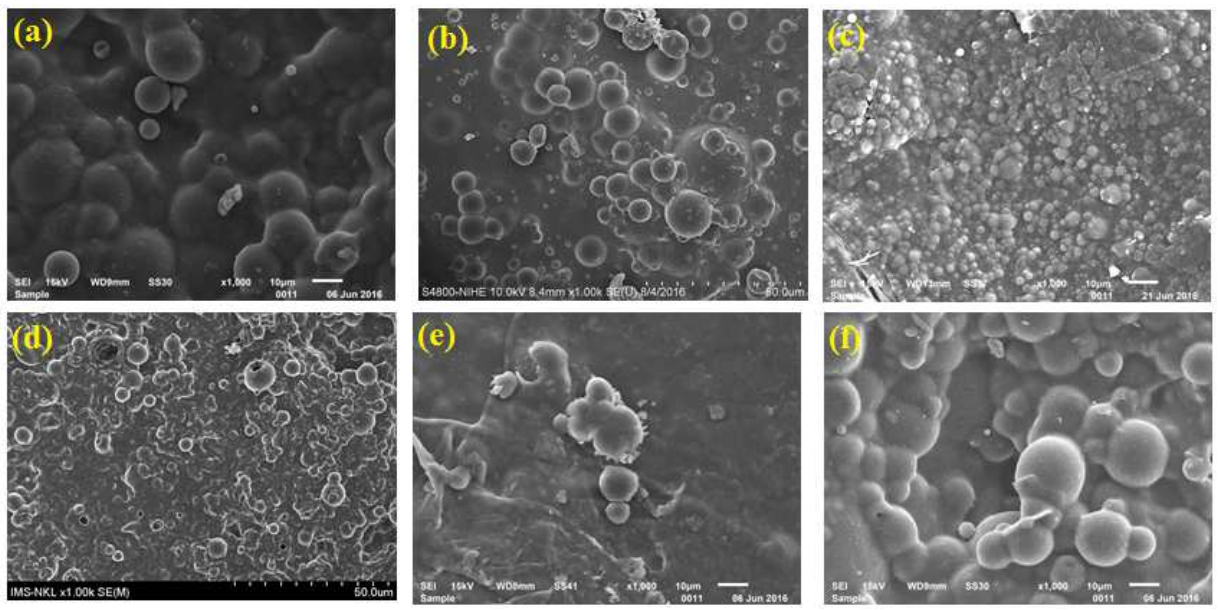

Figure 2. FESEM images of the PLA/CS/NIF films with various content of PEO at magnification of 1000 times: a) $0 \mathrm{wt} \%$ PEO, b) $2 \%$ PEO, c) $4 \mathrm{wt} \%$ PEO, d) $6 \mathrm{wt} \%$ PEO, e) $8 \mathrm{wt} \%$ PEO, f) $10 \mathrm{wt} \%$ PEO.
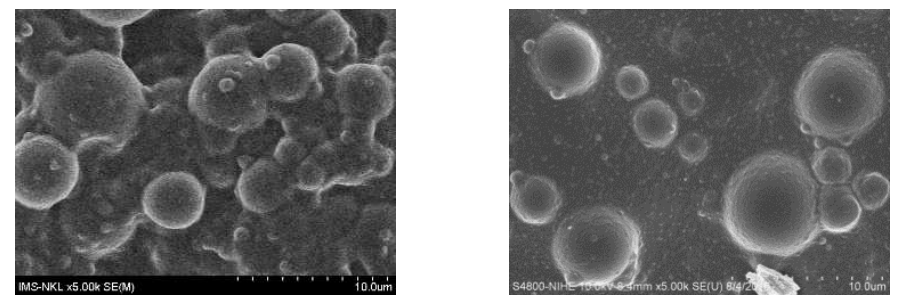

Figure 3. FESEM image of the PLA/CS/NIF/6 wt\% PEO films at magnification of 5000 times.

FESEM images of PLA/CS/NIF films with and without PEO after 24-hour drug release testing in $\mathrm{pH} 7.4$ solution are demonstrated in Figure 4. As results mentioned in our previous 
paper [6], the release of NIF from PLA/CS/NIF films is according to 2 steps: the first one is fast and the second one is slowly. From Figure 4, it is clear that PLA is hydrolyzed and left porous structure. In the FESEM image at 20000-time magnifications (Figure 4f), the holes with $50 \div 100$ $\mathrm{nm}$ size are appeared on the surface of CS particles. These holes are created by the drug release from the CS porous structure. It can be confirmed that NIF is absorbed and situated in the pores of the porous structure of CS and released from PLA/CS/NIF/PEO films.
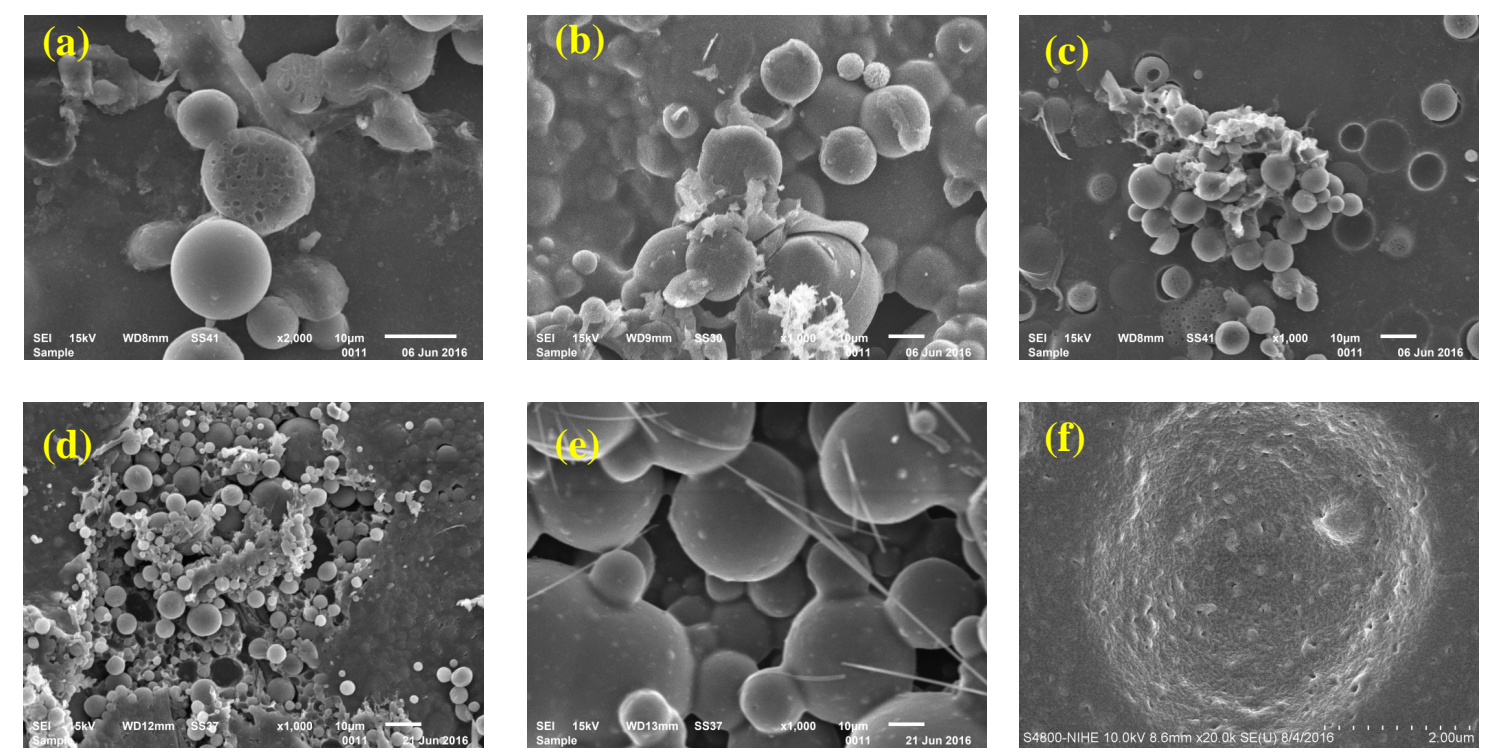

Figure 4. FESEM images of PLA/CS/NIF composite films at difference PEO content (a) 0 wt $\%$ PEO, (b) $4 \mathrm{wt} \%$ PEO, (c) $6 \mathrm{wt} \% \quad$ PEO, (d) $8 \mathrm{wt} \%$ PEO, (e) $10 \mathrm{wt} \%$ PEO after 24-hour drug release testing in $\mathrm{pH} 7.4$ solution (at 1000-time magnifications). (f) FESEM images of PLA/CS/NIF composite films with $6 \mathrm{wt} \%$ PEO content at 20000-time magnifications.

\subsection{Water absorption of PLA/CS/NIF films with and without PEO}

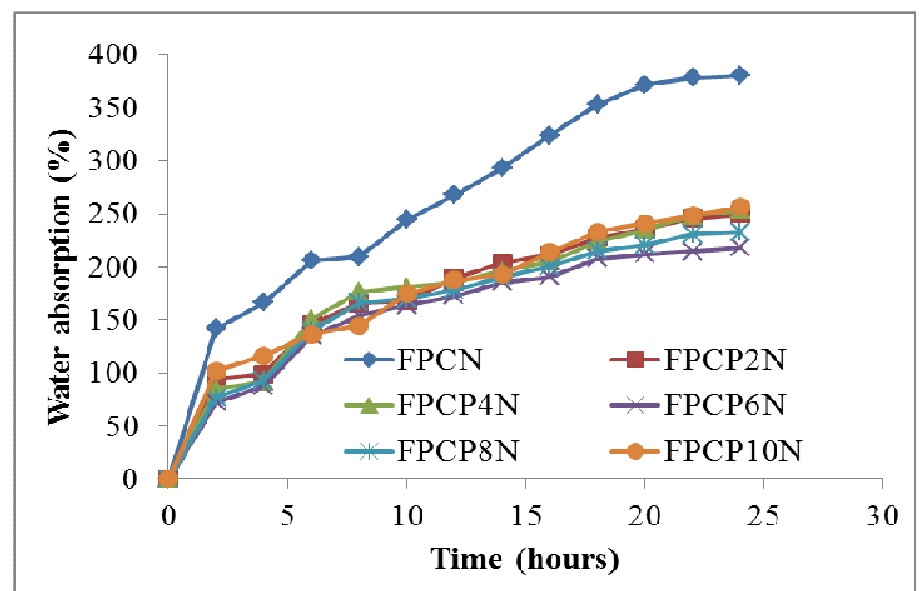

Figure 5. Water absorption of PLA/CS/NIF composite films with and without PEO. 
Water absorption is an important factor affected on the drug release from the polymer materials because drug is released and controlled through the swollen and hydrolyzed process of polymers. It corresponds to the water weight remained in the structure of films. For PLA/CS/NIF films, PLA has swelling and hydrolysis ability in water and swelling process is occurred before hydrolysis process. Water absorption of PLA/CS/NIF films with and without PEO is illustrated in Figure 5. It is increased significantly for the first 2 hours, reached $142 \%$ and then increased slowly. In contrast, the water absorption of the PLA/CS/NIF films is lower than that the sample without PEO and there is not much difference of the films with various content of PEO during investigation time.

In the presence of PEO, the interaction and dispersion between PLA, CS and NIF are improved clearly through hydrogen bonding and dipole-dipole interactions as mentioned in [16]. Among tested films, the FPCP6N and FPCP8N samples have the lowest water absorption due to their structure of PLA/CS/NIF films became more closely (see above 3.1. and 3.2). Therefore, the penetration of water molecules into the structure of films is limited and water absorption of the films using PEO is declined.

The water absorption value of PLA/CS/NIF films in this work is similar to that of chitosan/polylactide/Cloisite 30B composite materials carrying paclitaxel drug during the survey published by R. Nanda et al. [8].

\subsection{Weight loss of PLA/CS/NIF films with and without PEO in pH 7.4 solution}

Table 2 diplays the weight loss of PLA/CS/NIF films with and without PEO after 2, 7 , 14 and 28 days of immersing in $\mathrm{pH} 7.4$ solution. All investigated films have weight loss from $22.8 \%$ to $90.1 \%$ as increasing with immersing time. This indicates that PLA and NIF containing ester groups are hydrolyzed in $\mathrm{pH} 7.4$ solution. The weight loss of PLA/CS/NIF/PEO films (FPCPN) is lower than that of PLA/CS/NIF film without PEO (FPCN) at the same time of testing. It expresses that PLA/CS/NIF/PEO films have tighter structure causing the slower hydrolysis. The weight loss of FPCP6N and FPCP8N films is smaller than that of others. So, the suitable content of PEO for preparation of PLA/CS/NIF/PEO films is 6 and $8 \mathrm{wt} \%$.

Table 2. Weight loss of PLA/CS/NIF films with and without PEO immersed in $\mathrm{pH} 7.4$ solution.

\begin{tabular}{|c|c|c|c|c|}
\hline \multirow{2}{*}{ Samples } & \multicolumn{4}{|c|}{ Weight loss (\%) } \\
\cline { 2 - 5 } & 2 days & 7 days & 14 days & 28 days \\
\hline FPCN & 27.3 & 66.1 & 80.0 & 90.1 \\
\hline FPCP2N & 26.2 & 60.6 & 77.3 & 85.5 \\
\hline FPCP4N & 25.7 & 62.7 & 73.2 & 83.8 \\
\hline FPCP6N & 23.4 & 55.9 & 68.7 & 76.3 \\
\hline FPCP8N & 22.8 & 49.6 & 64.2 & 74.6 \\
\hline FPCP10N & 27.5 & 62.2 & 78.1 & 83.9 \\
\hline
\end{tabular}

\section{CONCLUSIONS}

PEO has an important role in significant improvement of the interaction between PLA, CS and NIF. The PEO content is affected on hydrophobicity and water absorption of the 
PLA/CS/NIF films. Using PEO in PLA/CS/NIF films, CS and NIF are dispersed more regularly with the smaller size in comparison to PLA/CS/NIF film. Especially, at $6 \%$ of PEO content, CS and NIF are dispersed with smallest size, about $1 \div 5 \mu \mathrm{m}$ and $50 \div 100 \mathrm{~nm}$, respectively. NIF is released from the PLA/CS/NIF/PEO films and left holes in the structure of these films immersed in $\mathrm{pH} 7.4$ solution. It confirms that the PLA/CS/NIF films with and without PEO are hydrolyzed in $\mathrm{pH} 7.4$ solution. The suitable content of PEO for preparation of PLA/CS/NIF/PEO films is 6 wt $\%$ and $8 \mathrm{wt} \%$.

Acknowledgements. This work has been financially supported by Vietnam National Foundation for Science and Technology Development (NAFOSTED, DT.NCCB-DHUD.2012-G/09) and Vietnam Academy of Science and Technology 2016.

\section{REFERENCES}

1. Gupta A. P., Kumar V. - New emerging trends in synthetic biodegradable polymers Polylactide: A critique, European Polymer Journal 43 (2007) 4053-4074.

2. No H. K., Meyers S. P., Prinyawiwatkul W. and Xu Z. - Applications of chitosan for Improvement of quality and shelf life of foods: A Review, Journal of Food Science 72 (5) (2007) 87-100 .

3. Singh D. K., Ray A. R. - Biomedical applications of chitin, chitosan, and their derivatives, J. M. S- Rev. Macromol. Chem. Phys. C 40 (1) (2000) 69-83.

4. Ramesh Babu V., Krishna Rao K. S. V., Yong Ill L. - Preparation and characterization of nifedipine - loaded cellulose acetate butyrate based microspheres and their controlled release behavior, Polym. Bull. 65 (2010) 157 - 167.

5. Rajan M., Raj V. - Formation and characterization of chitosan-polylacticacid-polyethylene glycol-gelatin nanoparticles: A novel biosystem for controlled drug delivery, Carbohydrate Polymers 98 (2013) 951-958.

6. Trang N. T. T., Chinh N. T., Giang N. V., Thanh D. T. M., Lam T. D., Hoang T. PLA/CS/Nifedipine nanocomposite films: Properties and the in-vitro release of nifedipine, Journal of Electronic Materials 45 (7) (2016) 3581-3590.

7. Thacharodi D., Panduranga R.K. - Collagen-chitosan composite membranes controlled transdermal delivery of nifedipine and propranolol hydrochloride, International Journal of Pharmaceutics 134 (1996) 239-241.

8. Nanda R., Sasmal A., Nayak P. L. - Preparation and characterization of chitosanpolylactide composites blended with Cloisite 30B for control release of the anticancer drug Paclitaxel, Carbohydrate Polymers 83 (2011) 988-994.

9. Sahoo S., Sasmal A., Nanda R., Phani A. R., Nayak P. L. - Synthesis of chitosanpolycaprolactone blend for control delivery of ofloxacin drug, Carbohydrate Polymers 79 (2010) 106-113.

10. Jeevitha D., Kanchana A. - Chitosan/PLA nanoparticles as a novel carrier for the delivery of anthraquinone: Synthesis, characterization and in vitro cytotoxicity evaluation, Colloids and Surfaces B: Biointerfaces 101 (2013) 126- 134.

11. Prabaharan M., Rodriguez-Perez M. A., de Saja J. A., Mano J. F. - Preparation and characterization of poly(D,L-lactic acid)/chitosan hybrid scaffold with drug release capability, J. Biomed Mater Res B Appl. Biomater. 81(2) (2007) 427-434. 
12. Constantin Edi.T. , Iulian S. - PLA/chitosan/keratin composites for biomedical applications, Materials Science and Engineering C 40 (2014) 242-247.

13. Dev A., Binulal N.S., Anitha A., Nair S.V., Fruike T., Tamura H., Jayakumar R. Preparation of poly(lactic acid)/chitosan nanopaticles for anti-HIV drug delivery applications, Carbohydrate Polymers 80 (2010) 833-838.

14. Chinh N.T., Cong D.V., Huynh M.D., Tuan V.M., Trang N.T.T., Mai T.T., Hoang T. Thermal property and morphology of poly lactic acid/chitosan composite containing nifedipine, Journal of Chemistry $\mathbf{5 3}$ (6) (2015) 706-712.

15. Chinh N. T., Trang N. T. T., Thanh D. T. M., Hang T. T. X., Giang N. V., Hoang T., Dung N.T., Quan P.M. - Thermal property, morphology and hydrolysis ability of poly(lactic acid)/chitosan nanocomposites using polyethylene oxide, Journal of Applied Polymer Science 132 (12) (2012) 3934-3939.

16. Nataly M. S., Ketlin C. G., Roberta B., Fernanda S. B., Marilene H.V., Rosane M. D. S. Poly (lactic acid)/chitosan fiber mats: Investigation of effects of the support on lipase immobilization, International Journal of Biological Macromolecules 72 (2015) 998-1004. 